\title{
1 Ecology and the diversification of reproductive strategies in
}

\section{2 viviparous fishes}

$4 \quad$ Michael Tobler ${ }^{1 *} \&$ Zachary Culumber ${ }^{2}$

$6 \quad{ }^{1}$ Division of Biology, Kansas State University, Manhattan, KS 66506, USA

$7 \quad{ }^{2}$ Department of Biological Sciences, University of Alabama in Huntsville, Huntsville, AL 35899,

8 USA

9

$10{ }^{*}$ Corresponding author: Michael Tobler, Division of Biology, Kansas State University, 106 Ackert

11 Hall, Manhattan, KS, USA; phone: +1-785-532-6652; email: tobler@ksu.edu 


\section{Abstract}

14 Shifts in life history evolution can potentiate sexual selection and speciation. However, we rarely

15 understand the causative links between correlated patterns of diversification or the tipping points

16 that initiate changes with cascading effects. We investigated livebearing fishes with repeated

17 transitions from pre-(lecithotrophy) to post-fertilization maternal provisioning (matrotrophy) to

18 identify the potential ecological drivers of evolutionary transitions in life history. Phylogenetic

19 comparative analyses across 94 species revealed that bi-directional evolution along the lecithotrophy-

20 matrotrophy continuum is correlated with ecology, supporting adaptive hypotheses of life history

21 diversification. Consistent with theory, matrotrophy was associated with high resource availability

22 and low competition. Our results suggest that ecological sources of selection contribute to the

23 diversification of female provisioning strategies in livebearing fishes, which have been associated

24 with macroevolutionary patterns of sexual selection and speciation.

26 Keywords: life history evolution | natural selection | phylogenetic comparative analysis | Poeciliidae 


\section{Introduction}

29 Evolutionary transitions in maternal provisioning strategies represent a primary axis of reproductive

30 life history variation in viviparous organisms (Wourms 1981; Blackburn 1992). Shifts from an

31 ancestral strategy of females providing all resources for embryonic development prior to fertilization

32 (lecithotrophy) to post-fertilization provisioning (matrotrophy) have been associated with the

33 evolution of complex physiological and morphological adaptations, including placental structures of

34 apposed maternal and embryonic tissues that facilitate nutrient transfer (Wooding and Burton 2008).

35 Matrotrophy and placentas have evolved repeatedly in viviparous animals, including multiple

36 invertebrate (Campiglia and Walker 1995; Hart et al. 1997; Korniushin and Glaubrecht 2003;

37 Korneva 2005; Ostrovsky et al. 2016) and vertebrate lineages (Steward and Blackburn 1988;

38 Wourms et al. 1988; Wake and Dickie 1998; Wildman et al. 2006). Transitions along the

39 lecithotrophy-matrotrophy continuum may have far reaching consequences, shaping the evolution

40 of other traits and patterns of biological diversification (Zeh and Zeh 2000; Coleman et al. 2009;

41 Pollux et al. 2014; Furness et al. 2015; Furness et al. 2019). What evolutionary forces shape the

42 evolution of matrotrophy, however, remains unclear (Pollux et al. 2009).

There are two, non-mutually exclusive hypotheses that view matrotrophy as an adaptation to

44 ecological sources of selection: (1) Matrotrophy has been hypothesized to reduce locomotor costs

45 associated with pregnancy (Magnhagen 1991; Shaffer and Formanowicz 1996; Miles et al. 2000).

46 Specifically, lecithotrophic females are expected to suffer from impaired locomotion throughout

47 gestation, while matrotrophic females with initially small embryos should avoid such costs at least in

48 early stages of pregnancy (Miller 1975; Thibault and Schultz 1978). (2) Resource availability may

49 shape evolution along the lecithotrophy-matrotrophy continuum. Lecithotrophy is expected to be

50 adaptive in environments with fluctuating resource availability (Thibault and Schultz 1978), whereas 
matrotrophy theoretically maximizes reproductive output when resource availability is high and stable (Trexler and DeAngelis 2003). hypotheses about the evolution of reproductive strategies at micro- and macroevolutionary scales (Evans et al. 2011). Poeciliids have undergone remarkable diversification in levels of postfertilization maternal provisioning, with independent origins of matrotrophy in different clades (Reznick et al. 2002; Pollux et al. 2009; Pollux et al. 2014). Research on poeciliid fishes has been instrumental for the advancement of our theoretical and empirical understanding of matrotrophy

59 (Thibault and Schultz 1978; Pollux et al. 2009; Pires et al. 2011), and there is evidence supporting various predictions of both adaptive hypotheses of matrotrophy evolution. Pregnancy in poeciliids has been shown to be associated with locomotor costs (Plaut 2002; Ghalambor et al. 2004; Quicazan-Rubio et al. 2019), and there is evidence that matrotrophy increases streamlining (Fleuren et al. 2018) and may be favored in high predation environments requiring efficient escape responses

64 (Gorini-Pacheco et al. 2017; Hagmayer et al. 2020). At the same time, post-fertilization maternal provisioning also responds to resource availability and female condition (Trexler 1997; MarshMatthews and Deaton 2006; Hagmayer et al. 2018), and matrotrophy is associated with significant costs when resources fluctuate (Pollux and Reznick 2011). However, most empirical tests of the potential adaptive function of matrotrophy have been conducted at microevolutionary scales and using just a small number of species, leaving questions about the generalizability of past findings.

71 spanning the family Poeciliidae. Species in this family are broadly distributed throughout the

72 Americas, found in a wide variety of ecological contexts, and have a well-resolved phylogeny (Meffe 73 and Snelson 1989; Hrbek et al. 2007; Reznick et al. 2017), facilitating comparative analyses that

74 contrast hypotheses about the evolutionary origins of matrotrophy. To do so, we first characterized 
75 the evolutionary dynamics of matrotrophy evolution and then used phylogenetic path analysis to test

76 for correlations between ecological variables and variation in matrotrophy.

\section{Methods}

\section{Taxon sampling and phylogenetic framework}

80 Our analyses included 94 species (Table S1), encompassing representatives of all major genera in the

81 family Poeciliidae. These species span a geographic range from the eastern United States south to

82 Argentina, including Caribbean islands (Figure S1). The phylogenetic framework used for analyses

83 was established by previous studies with similar taxon sampling (Pollux et al. 2014; Culumber and

84 Tobler 2017). In brief, sequences for six mitochondrial (12S, COI, CytB, ND2, $t R N A v a l u$, and

85 tRNAleu) and 11 nuclear genes (Beta Actin, CCND1, ENC1, GLYT, MYH6, RAG1, Rhodopsin, RPS7,

86 SH3PX3, T36, and XSRC) were obtained from GenBank, aligned, and maximum likelihood

87 phylogenetic analysis was conducted using RAxML-HPC version 8 (Stamatakis 2014) on the

88 CIPRES computer cluster (San Diego State University, San Diego, CA, USA). The resulting best

89 scoring tree was highly consistent with previously published phylogenetic hypotheses for the family

90 Poeciliidae (Hrbek et al. 2007; Pollux et al. 2014; Reznick et al. 2017). Phylogenetic trees were time

91 calibrated with three calibration points spanning the depth of the phylogeny (see Culumber and

92 Tobler 2017 for details), including a primary fossil calibration associated with the split separating the

93 outgroup (Fundulus) from all poeciliids (55-99 Ma; Santini et al. 2009) and a secondary fossil

94 calibration separating Heterandria formosa from the genus Poecilia (9.3-19 Ma; Ho et al. 2016). In

95 addition, the formation of Laguna de Catemaco (Mexico), a crater lake with several endemic species,

96 was used as a constraint on the age of the endemic Poeciliopsis catemaco (0.5-2.0 Ma; Mateos et al.

97 2002). Even though bootstrap support values of the best scoring tree were generally strong, 
98 phylogenetic comparative methods described below were conducted across 250 trees drawn at

99 random from the bootstrap replicates to account for phylogenetic uncertainty.

\section{Quantifying matrotrophy and sexual selection}

102 Matrotrophy levels for all species included in the analysis were obtained from previously published

103 studies (Pollux et al. 2014; Olivera-Tlahuel et al. 2015). The extent of post-fertilization maternal

104 provisioning was quantified using the matrotrophy index (MI, ln-transformed for all analyses), which

105 is the ratio of offspring mass at birth to the mass of the egg at fertilization (Reznick et al. 2002;

106 Pollux et al. 2009). Offspring of lecithotrophic species typically lose $25-55 \%$ of the initial egg mass

107 during development $(\mathrm{MI}<0.75)$, while continuous nutrient transfer from mother to offspring

108 during gestation in matrotrophic species leads to MI > 1 (Reznick et al. 2002; Pollux et al. 2009).

\section{Evolutionary dynamics of maternal provisioning strategies}

111 To characterize the evolutionary dynamics of matrotrophy evolution, we conducted ancestral state

112 reconstructions of MI using PHYTOOLS (Revell 2012). To evaluate the directionality of matrotrophy

113 evolution (increases $v s$. decreases in MI) between each node and its descendants, we extracted trait

114 reconstructions for each node of the tree and calculated $\Delta \mathrm{MI}$ as the observed (tip) or inferred (node)

115 matrotrophy values subtracted from the values of its most recent ancestral node.

117 Identifying ecological correlates of variation in maternal provision strategies

118 To test the competing hypotheses for matrotrophy evolution, we assembled a set of relevant

119 environmental predictor variables. According to the locomotor hypothesis, matrotrophy should be

120 associated with environments that favor high locomotor performance, such as habitats with high

121 predation pressure or fast water currents (Reznick et al. 2007; Gorini-Pacheco et al. 2017). Hence, 
122 predictor variables included metrics of hydrology (based on the topography of each species' range)

123 and predation (number of predatory fish species overlapping each species' range). According to the

124 resource availability hypothesis, matrotrophy should be associated with environments where

125 resources are abundant and competition is low (Trexler and DeAngelis 2003). Hence, we quantified

126 climate (including estimates of temporal variability in temperature and precipitation patterns in each

127 species' range), average net primary productivity (NPP), and competition (number of poeciliid

128 species overlapping each species' range). The potential effects of different environmental variables

129 on variation in maternal provisioning strategies was evaluated with phylogenetic path analysis as

130 outlined below.

Quantifying bydrology, climate, and net primary productivity

133 Assembly of hydrological and climate variables associated with each species' range was based on

134 georeferenced occurrence points. We obtained 73,398 locality points from multiple sources

135 (http://fishnet2.net/, http://gbif. org/, primary literature), representing the known distributions for

136 all 94 species included in our study. We first removed duplicate points and retained a maximum of

137100 randomly sampled localities within the native range of each species, which is sufficient to

138 capture environmental variation even in wide-ranging species (van Proosdij et al. 2015). We further

139 verified that all data points for a given species were at least $1 \mathrm{~km}$ apart to match the spatial

140 resolution of environmental data. Any locality that did not meet this criterion was either removed

141 for species with $<100$ localities or replaced by another randomly drawn locality for species with

$142>100$ localities. For all locality records, we then extracted values for three hydrological variables

143 (elevation, slope and compound topographic index; Hydro1k:

144 https://lta.cr.usgs.gov/HYDRO1K/), 19 climatic variables (Worldclim: http://worldclim.org/), and

145 an estimate of net primary productivity (https://pdaac.usgs.gov/) at a spatial resolution of $\sim 1 \mathrm{~km}^{2}$ 
146 (30 arcsec) in ArcMap version 10.2.2 (ESRI Inc, Redlands, CA, USA). For each species, we

147 calculated the median value for all 23 variables. Climatic and hydrological variables were then

148 subjected to separate phylogenetic principal component analyses (pPCA) using a correlation matrix,

149 as implemented in the PHYTOOLS package in R (Revell 2012). In addition to the estimate of net

150 primary productivity associated with each species' range, we retained two PPCA axes accounting for

$15176 \%$ of variation in hydrology (Table S2) and three pPCA axes accounting for $81 \%$ of variation in

152 climate (Table S3).

153

Quantifying competition and predation

155 Quantifying the actual biotic interactions for a large number of species distributed across the vast

156 geographic scale included in this study is virtually impossible. Hence, we developed two simple,

157 objectively quantifiable metrics to approximate levels of competition and predation. We assumed

158 that competitive interactions for the focal species primarily occur with other species of the family

159 Poeciliidae (Alberici da Barbiano et al. 2010; Torres-Dowdall et al. 2013) and that the intensity of

160 competition is a function of the number of coexisting species. Hence, we first analyzed overlap of

161 distributional ranges to characterize patterns of sympatry (defined as range overlap values greater

162 than zero; Weber et al. 2016). We created geo-referenced distributional range maps for each species

163 by generating a convex hull around each species' known occurrence points (see above) using

164 ArcMap. The resulting species-specific distributions were then intersected to determine the total

165 number of competitor species exhibiting a range overlap with a focal species. Similarly, we created a

166 metric estimating the levels of predation by determining the total number of piscivorous fish species

167 exhibiting a range overlap with each focal species. To do so, we obtained 271,148 locality points

168 (http://gbif. org/) of 7,170 species across 1,602 genera and 26 families in the superclass

169 Osteichthyes that coincide with the distribution of poeciliids. Since distributional polygons of some 
170 focal species overlapped with marine habitats (particularly in poeciliid species occurring along the

171 Gulf of Mexico and in both island and mainland localities), we first removed species primarily

172 inhabiting marine environments as well as non-native species, retaining 5,019 native freshwater

173 species in 853 genera (Table S4). To identify potential predators, we conducted a genus-level

174 literature search of dietary habits using relevant monographs (Greenfield and Thomerson 1997;

175 Bussing 1998; Boschung et al. 2004; Miller et al. 2005; van der Sleen and Albert 2017), supplemented

176 by the primary literature when necessary. We retained 131 genera that included species with evidence

177 for piscivory (867 species; 73,421 locality points). Values for the number of competitors and

178 predators were square-root-transformed prior to analyses.

180 Analytical framework.

181 We investigated hypotheses about the hierarchical relationships among abiotic and biotic

182 environmental factors and matrotrophy using phylogenetic path analysis as implemented in the $\mathrm{R}$

183 package PHYLOPATH (van der Bij 2018). We developed 18 models based on a priori hypotheses

184 about the effects of hydrology, climate, NPP, competition, predation, and interactions between

185 predictor variables relevant in the context of the locomotor cost and resource limitation hypotheses

186 of matrotrophy evolution (Figure S2). As with other phylogenetic analyses described above, path

187 analyses were run across 250 random trees. PHYLOPATH implements model selection with covariance

188 inflation criterion, CIC $_{C}$ (Rodriguez 2005). Models with an average $\Delta$ CIC $_{C}<2$ were considered

189 equally supported (Burnham and Anderson 2002). Joint effects of net primary productivity and

190 competition were visualized using non-parametric thin-plate spline regression to create a surface of

191 matrotrophy variation (Arnold 2003). Estimation of matrotrophy surfaces was performed using the

192 FIELDS package in $\mathrm{R}$, with smoothing parameter $\lambda=0.005$ (Nychka et al. 2007). 


\section{Results}

195

196

197

198

199 95\% CI $=0.022-0.025$; secondary model: $r=0.023,95 \%$ CI $=0.021-0.024)$. Simultaneously

\section{Evolutionary dynamics of matrotrophy evolution during diversification of poeciliid fishes}

Ancestral state reconstructions (ASR) were used to compare inferred ancestral states of matrotrophy to variation in matrotrophy observed in extant taxa (Figure 1A). ASRs across 250 trees

demonstrated that lecithotrophy is not the ancestral provisioning strategy (Figure 1B). The inferred ancestral state was clearly toward the matrotrophic end of the spectrum (with a net weight gain during development) and distinctly above the levels of post-fertilization provisioning observed in most extant taxa (Figure 1B). This does not mean that matrotrophy evolved prior to lecithotrophy, but rather that the common ancestor of extant poeciliids had already evolved some degree of postfertilization provisioning. Examining the direction of shifts in post-fertilization provisioning strategies between all nodes and their descendants revealed that reductions of post-fertilization provisioning were just as common as increases in matrotrophy (Figure 1C).

\section{Ecological correlates of matrotrophy evolution}

We contrasted a series of hypotheses about the hierarchical relationships among different abiotic and biotic environmental variables and matrotrophy using phylogenetic path analysis. Model selection identified two models with average $\Delta \mathrm{CIC}_{\mathrm{c}}<2$ across the 250 trees (Figure S3), including NPP, competition, and predation as predictor variables for variation in MI (Figure 2A). Both models indicated that NPP positively correlated with competition $(r=0.325,95 \% \mathrm{CI}=0.324-0.326$ for both supported models), which in turn was negatively correlated with matrotrophy (top model: $r=$ 0.030, 95\% CI $=-0.034--0.025$; secondary model: $r=-0.077,95 \% \mathrm{CI}=-0.081--0.075)$. In addition, there was a positive relationship between NPP and matrotrophy (top model: $r=0.024$, visualizing the effects of NPP and competition on matrotrophy indicated that high levels of 
matrotrophy occurred when NPP was high and competition was low (Figure 2B). In the best

supported model, NPP was also positively correlated with predation $(r=0.184,95 \%$ CI $=0.183-$

0.185), which in turn was negatively correlated with matrotrophy $(r=-0.082,95 \% \mathrm{CI}=-0.087--$

0.078). Notably, however, the directionality of the relationship between predation and matrotrophy indicating that ecology has played a role in matrotrophy evolution.

\section{Discussion}

228 Shifts from pre- to post-fertilization maternal provisioning represent a major axis of life history

229 evolution in viviparous organisms (Wourms 1981; Blackburn 1992). Even though life history

230 evolution is generally assumed to progress from oviparity to lecithotrophic viviparity to

231 matrotrophic viviparity (e.g., Furness et al. 2015), our analyses indicated that decreases in levels of

232 matrotrophy were just as common as increases. Such bi-directional evolution along the

233 lecithotrophy-matrotrophy continuum has been documented in other viviparous taxa (e.g., Dulvy

234 and Reynolds 1997; Reznick et al. 2007) and parallels secondary losses of obligate viviparity in fishes

235 and reptiles (Parenti et al. 2010; Recknagel et al. 2018). The standard model of linear life history

236 evolution therefore needs reevaluation to acknowledge that the evolution of these traits is more

237 complex and dynamic than generally appreciated.

239 bi-directional evolution along the lecithotrophy-matrotrophy continuum. Phylogenetic path analysis

240 identified three biotic variables that were associated with variation in matrotrophy (resource

241 availability, competition, and predation), all of which are well-documented drivers of life history 
242 diversification in animals (Stearns 1976; Martin 1995; Wilson 2014). However, the relationship

243 between matrotrophy and predation was opposite to the predictions of the locomotor hypothesis,

244 even though microevolutionary analyses have indicated that matrotrophy can be favored in high

245 predation environments requiring efficient escape responses (Gorini-Pacheco et al. 2017; Hagmayer

246 et al. 2020). Instead, our results provided support for the resource availability hypothesis,

247 demonstrating that high levels of matrotrophy coincided with low competition and high resource

248 availability. This finding is consistent with the predictions of theoretical models, which predict

249 increases in matrotrophy in environments with abundant and stable resources (Trexler and

250 DeAngelis 2003), and empirical observations (Pollux and Reznick 2011). In addition, experimental

251 studies have shown that maternal provisioning strategies in poeciliids respond to resource availability

252 and female body condition (Trexler 1997; Marsh-Matthews and Deaton 2006; Hagmayer et al. 2018).

253 Our results largely align with previous theoretical and empirical studies; however, there are

254 some caveats that warrant additional consideration. Most importantly, it remains to be tested

255 whether metrics of resource availability, competition, and predation used here to facilitate

256 continental-scale analyses accurately reflect selective regimes experienced by different species. The

257 challenges of quantifying complex variation in biotic interactions across the vast spatial and

258 phylogenetic scales covered in this study highlights the need for microevolutionary analyses and

259 experimental approaches with a broader taxon sampling that allow for a better understanding of

260 causal relationships (Culumber and Tobler 2018). For example, while there is experimental evidence

261 for the fitness costs of matrotrophy under fluctuating resource conditions (Pollux and Reznick

262 2011), we still lack any empirical evidence indicating that matrotrophy provides fitness benefits over

263 lecithotrophy under high and stable resource conditions (Pollux et al. 2009). In addition, it remains

264 to be experimentally tested how resource availability and competition potentially interact in

265 determining the success of different maternal provisioning strategies, especially because resource 
266 stress and competitive interactions may have non-additive effects (Hart and Marshall 2013; van

267 Egmond et al. 2018).

\section{Conclusions}

270 The role of ecological sources of selection as key drivers in life history evolution is well established

271 at microevolutionary scales (Partridge and Harvey 1988; Reznick et al. 1990). Our study revealed that

272 ecology also correlates with maternal provisioning strategies at macroevolutionary scales, suggesting

273 that adaptation to resource availability and competition could explain life history diversification in

274 livebearing fishes and potentially in other viviparous taxa (Wourms 1981; Blackburn 1992). Our

275 findings do not exclude the possibility that other evolutionary forces are also at play. For example,

276 variation in maternal provisioning strategies has been associated with parent-offspring conflict

277 (Schrader and Travis 2008; Ala-Honkola et al. 2011; Pollux et al. 2014), potentially leading to

278 antagonistic coevolution between maternal and embryonic traits that impact nutrient transfer during

279 pregnancy (Crespi and Semeniuk 2004; Furness et al. 2015). Further studies will consequently need

280 to explore how ecological sources of selection might interact with parent-offspring conflict to shape

281 the evolution of matrotrophy. Disentangling the drivers of matrotrophy evolution will be particularly

282 interesting because changes in female provisioning strategies can potentiate (or impede) sexual

283 selection and speciation (Zeh and Zeh 2000; Zeh and Zeh 2008) and explain broad-scale patterns of

284 diversification in viviparous taxa (Furness et al. 2019).

285

\section{Data Accessibility}

287 Data used to conduct this study will be archived on Dryad upon acceptance of the manuscript.

\section{Acknowledgments}


This research was supported by the National Science Foundation (IOS-1557860 to MT). We thank members of the K-State Evolution Journal Club for constructive feedback on the manuscript.

\section{References}

294 Ala-Honkola, O., E. Friman, and K. Lindström. 2011. Costs and benefits of polyandry in a placental poeciliid fish Heterandria formosa are in accordance with the parent-offspringconflict theory of placentation. Journal of Evolutionary Biology 24:2600-2610.

Alberici da Barbiano, L., J. Waller, and C. Gabor. 2010. Differences in competitive efficiency

Arnold, S. J. 2003. Performance surfaces and adaptive landscapes. Integrative and Comparative Biology 43:367-375.

Blackburn, D. G. 1992. Convergent evolution of viviparity, matrotrophy, and specializations for fetal nutrition in reptiles and other vertebrates. American Zoologist 32:313-321. Washinton, D. C.

Burnham, K. P. and D. R. Anderson. 2002. Model selection and multimodel inference: a practical information-theoretic approach. Springer, New York, NY. 
313 Coleman, S. W., A. Harlin-Cognato, and A. G. Jones. 2009. Reproductive isolation, reproductive

314 mode, and sexual selection: empirical tests of the viviparity-driven conflict hypothesis.

$315 \quad$ American Naturalist 173:291-303.

316 Crespi, B. and C. Semeniuk. 2004. Parent-offspring conflict in the evolution of vertebrate

317 reproductive mode. American Naturalist 163:635-653.

318 Culumber, Z. W. and M. Tobler. 2017. Sex-specific evolution during the diversification of live-

Culumber, Z. W. and M. Tobler. 2018. Correlated evolution of thermal niches and functional bearing fishes. Nature Ecology \& Evolution 1:1185-1191.

Dulvy, N. K. and J. D. Reynolds. 1997. Evolutionary transitions among egg-laying, live-bearing and maternal inpurts in sharks and rays. Proceedings of the Royal Society B 264:1309-1315.

Evans, J. P., A. Pilastro, and I. Schlupp, eds. 2011. Ecology and Evolution of Poeciliid Fishes. The University of Chicago Press, Chicago, IL.

Fleuren, M., E. M. Quicazan-Rubio, J. L. van Leeuwen, and B. J. A. Pollux. 2018. Why do placentas evolve? Evidence for a morphological advantage during pregnancy in live-bearing fish. PLoS ONE 13:e0195976.

Furness, A. I., K. R. Morrison, T. J. Orr, J. D. Arendt, and D. N. Reznick. 2015. Reprodictive mode and the shifting arenas of evolutionary conflict. Annals of the New York Academy of

332 Furness, A. I., B. J. A. Pollux, R. W. Meredith, M. S. Springer, and D. N. Reznick. 2019. How conflict shapes the evolution in poeciliid fishes. Nature Communications 10:3335.

334 Ghalambor, C. K., D. N. Reznick, and J. A. Walker. 2004. Constraints on adaptive evolution: the functional trade-off between reproduction and fast-start swimming performance in the Trinidadian guppy (Poecilia reticulata). American Naturalist 164:38-50. 
Gorini-Pacheco, B., E. Zandonà, and R. Mazzoni. 2017. Predation effects on matrotrophy, superfetation and other life history traits in Phalloceros harpagos. Ecology of Freshwater Fish $27: 442-452$.

Greenfield, D. W. and J. E. Thomerson. 1997. Fishes of the continental waters of Belize. The University Press of Florida, Gainesville.

Hagmayer, A., A. I. Furness, D. N. Reznick, M. L. Dekker, and B. J. A. Pollux. 2020. Predation risk shapes the degree of placentation in natural populations of live-bearing fish. Ecology Letters 23:831-840.

Hagmayer, A., A. I. Furness, D. N. Reznick, and B. J. A. Pollux. 2018. Maternal size and body condition predict the amount of post-fertilization maternal provisioning in matrotrophic fish. Ecology and Evolution 8:12386-12396.

Hart, M. W., M. Byrne, and M. J. Smith. 1997. Molecular phylogenetic analysis of life-history evolution in asterinid starfish. Evolution 51:1848-1861.

Hart, S. P. and D. J. Marshall. 2013. Environmental stress, facilitation, competition, and coexistence. Ecology 94:2719-2731.

Ho, A. F. L. C., C. L. Pruet, and J. Lin. 2016. Phylogeny and biogeography of Poecilia (Cypinodontiformes: Poeciliinae) across Central and South America based on mitochondrial and nuclear DNA markers. Molecular Phylogenetics and Evolution 101:32-45.

Hrbek, T., J. Seckinger, and A. Meyer. 2007. A phylogenetic and biogeographic perspective on the evolution of poeciliid fishes. Molecular Phylogenetics and Evolution 43:986-998.

Korneva, Z. V. 2005. Placental type interactions and evolutionary trends ofdevelopment of uterus in cestodes. Journal of Evolutionary Biochemistry and Physiology 41:552-560. 
Korniushin, A. V. and M. Glaubrecht. 2003. Novel reproductive modesin freshwater clams: brooding and larval morphology in southeast Asian taxa of Corbicula (Mollusca, Bivalvia, Corbiculidae). Acta Zoologica 84:293-315.

Magnhagen, C. 1991. Predation risk as a cost of reproduction. Trends in Ecology and Evolution 6:183-185.

Marsh-Matthews, E. and R. Deaton. 2006. Resources and offspring provisioning: A test of the Trexler-DeAngelis model for matrotrophy evolution. Ecology 87:3014-3020.

Martin, T. E. 1995. Avian life history evolution in relation to nest sites, nest predation, and food. Ecological Monographs 65:101-127.

Mateos, M., O. I. Sanjur, and R. C. Vrijenhoek. 2002. Historical biogeography of the livebearing fish genus Poeciliopsis (Poeciliidae: Cyprinodontiformes). Evolution 56:972-984.

Meffe, G. K. and F. F. Snelson. 1989. An ecological overview of poeciliid fishes. Pp. 13-31 in G. K. Meffe, and F. F. Snelson, eds. Ecology and evolution of lifebearing fishes (Poeciliidae). Prentice Hall, New Jersey.

Miles, D. B., B. Sinervo, and W. A. Frankino. 2000. Reproductive burden, locomotor performance, and the cost of reproduction in free ranging lizards. Evolution 54:1386-1395.

Miller, R. R. 1975. Five new species of Mexican poeciliid fishes of the genera Poecilia, Gambusia, and Poeciliopsis. Occasional Papers of the Museum of Zoology, University of Michigan 672:1-44.

Miller, R. R., W. Minckley, and S. Norris. 2005. Freshwater fishes of Mexico. University of Chicago Press, Chicago.

Nychka, D., R. Furrer, J. Paige, and S. Sain. 2007. fields: tools for spatial data. R package version 9.6.

Olivera-Tlahuel, C., A. G. Ossip-Klein, H. S. Espinosa-Pérez, and J. J. Zúñiga-Vega. 2015. Have superfetation and matrotrophy facilitated the evolution of larger offspring in poeciliid fishes? Biological Journal of the Linnean Society 116:787-804. 
Ostrovsky, A. N., S. Lidgard, D. P. Gordon, T. Schwaha, G. Genikhovich, and A. V. Ereskovsky. 2016. Matrotrophy and placentation in invertebrates: a new paradigm. Biological Reviews 91:673-711.

Parenti, L. R., F. L. LoBNostro, and H. Grier. 2010. Reproductive histology of Tomeurus gracilis Eigenmann, 1909 (Teleostei: Atherinomorpha: Poeciliidae) with comments on evolution of viviparity in atherinomorph fishes. Journal of Morphology 27:1399-1406.

Partridge, L. and P. H. Harvey. 1988. The ecological context of life history evolution. Science 241:1449-1455.

Pires, M. N., A. I. Banet, B. J. A. Pollux, and D. N. Reznick. 2011. Variation and evolution of reproductive strategies. Pp. 28-37 in J. P. Evans, A. Pilastro, and I. Schlupp, eds. Ecology and Evolution of Poeciliid Fishes. University of Chicago Press, Chicago, IL.

Plaut, I. 2002. Does pregnancy affect swimming performance in female mosquitofish, Gambusia affinis? Functional Ecology 16:290-295.

Pollux, B., M. Pires, A. Banet, and D. Reznick. 2009. Evolution of placentas in the fish family Poeciliidae: an empirical study of macroevolution. Annual Review of Ecology Evolution and Systematics 40:271-289.

Pollux, B. J. A., R. W. Meredith, M. S. Springer, T. Garland, and D. N. Reznick. 2014. The evolution of the placenta drives a shift in sexual selection in livebearing fish. Nature 513:233-236.

Pollux, B. J. A. and D. N. Reznick. 2011. Matrotrophy limits a female's ability to adaptively adjust offspring size and fecundity in fluctuating environments. Functional Ecology 25:747-756.

Quicazan-Rubio, E. M., C. L. van Leeuwen, K. van Manen, M. Fleuren, B. J. A. Pollux, and E. J. Stamhuis. 2019. Coasting in live-bearing fish: the drag penalty of being pregnant. Journal of the Royal Society Interface 16:20180714. 
Recknagel, H., N. A. Kamenos, and K. R. Elmer. 2018. Common lizards break Dollo's law of irreversibility: genome-wide phylogenomics support a single origin of viviparity and reevolution of oviparity. Molecular Phylogenetics and Evolution 127:579-588.

Revell, L. J. 2012. phytools: an R package for phylogenetic comparative biology (and other things). Methods in Ecology and Evolution 3:217-223.

Reznick, D., H. Bryga, and J. A. Endler. 1990. Experimentally induced life-history evolution in a natural population. Nature 346:357-359.

Reznick, D., M. Mateos, and M. Springer. 2002. Independent origins and rapid evolution of the placenta in the fish genus Poeciliopsis. Science 298:1018-1020.

Reznick, D., R. Meredith, and B. B. Collette. 2007. Independent evolution of complex life history adaptation in two families of fishes, live-bearing halfbeaks (Zenarchopteridae, Beloniformes) and Poeciliidae (Cyprinodontiformes). Evolution 61:2570-2583.

Reznick, D. N., A. I. Furness, R. W. Meredith, and M. S. Springer. 2017. The origin and biogeographic diversification of fishes in the family Poeciliidae. PLoS ONE 12:e0172546.

Rodriguez, C. C. 2005. The ABC of model selection: AIC, BIC, and the new CIC. AIP Conference Proceedings 803:80-87.

Santini, F., L. J. Harmon, G. Carnevale, and M. E. Alfaro. 2009. Did genome duplication drive the origin of teleosts? A comparative study of diversification in ray-finned fishes. BMC Evolutionary Biology 9:1.

Schrader, M. and J. Travis. 2008. Testing the viviparity-driven-conflict hypothesis: parent-offspring conflict and the evolution of reproductive isolation in a poeciliid fish. American Naturalist 172:806-817.

Shaffer, L. R. and D. R. Formanowicz. 1996. A cost of viviparity and parental care in scorpions: reduced sprint speed and behavioral compensation. Animal Behavior 51:1017-1024. 
Stamatakis, A. 2014. RAxML version 8: a tool for phylogenetic analysis and post-analysis of large phylogenies. Bioinformatics 30:1312-1313.

Stearns, S. C. 1976. Life-history tactics: a review of ideas. Quarterly Review of Biology 51:3-47.

Steward, J. R. and D. G. Blackburn. 1988. Reptilian placentation: structural diversity and terminology. Copeia 1988:839-852.

Thibault, R. E. and R. J. Schultz. 1978. Reproductive adaptions among viviparous fishes gradient. Biological Journal of the Linnean Society 108:790-805.

Trexler, J. C. 1997. Resource availability and plasticity in offspring provisioning: Embryo nourishment in sailfin mollies. Ecology 78:1370-1381.

Trexler, J. C. and D. L. DeAngelis. 2003. Resource allocation in offspring provisioning: An evaluation of the conditions favoring the evolution of matrotrophy. American Naturalist

van der Bijl, W. 2018. phylopath: easy phylogenetic path analysis in R. PeerJ 6:e4718.

van der Sleen, P. and J. S. Albert, eds. 2017. Field guide to the fishes of the Amazon, Orinoco, and Guianas. Princeton University Press, Princeton. community are independent of food availability but driven by complementarity effects. Ecology and Evolution 8:3086-3097.

van Proosdij, A. S., M. S. Sosef, J. J. Wieringa, and N. Raes. 2015. Minimum required number of specimen records to develop accurate species distribution models. Ecography. 
454 Wake, M. H. and R. Dickie. 1998. Oviduct structure and function and reproductive modes in amphibians. Journal of Experimental Zoology 282:477-506.

456 Weber, M. G., L. Mitko, T. Eltz, and S. R. Ramírez. 2016. Macroevolution of perfume signalling in orchid bees. Ecology Letters 19:1314-1323.

458 Wildman, D. E., C. Chen, O. Erez, L. I. Grossman, M. Goodman, and R. Romero. 2006. Evolution of the mammalian placenta revealed by phylogenetic analysis. Proceedings of the National Academy of Sciences USA 103:3203-3208.

461 Wilson, A. J. 2014. Competition as a source of constraint on life history evolution in natural populations. Heredity 112:70-78.

463 Wooding, P. and G. Burton. 2008. Comparative placentation: structures, functions and evolution. 464 Springer-Verlag, Berlin.

465 Wourms, J. P. 1981. Viviparity: the maternal-fetal relationship in fishes. American Zoologist 21:473-

466 515.

467 Wourms, J. P., B. D. Grove, and J. L. Lombardi. 1988. The maternal-fetal relationship in viviparous 468 fishes. Pp. 2-134 in W. S. Hoar, ed. Fish Physiology. Academic Press, London. 
A

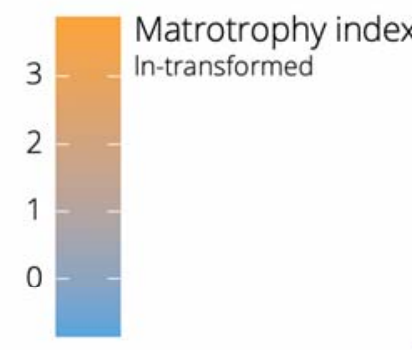

(1)
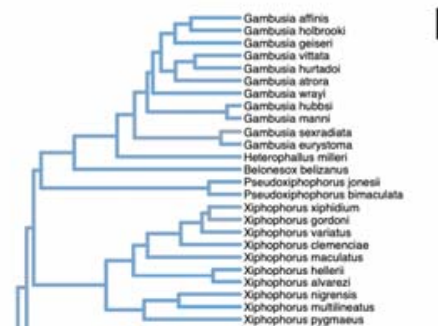

B.

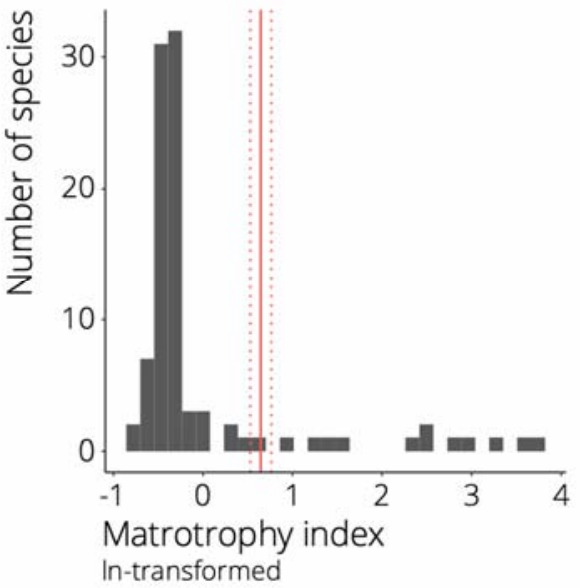

C

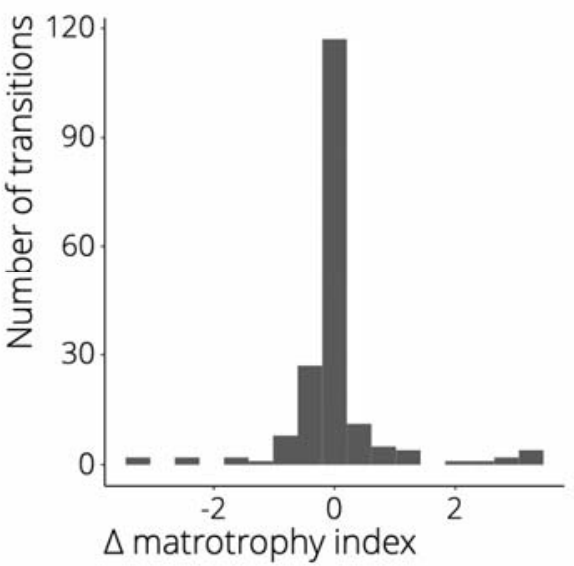

475 Figure 1. A. Best-scoring maximum likelihood tree of 94 species in the family Poeciliidae. The

476 ancestral state reconstruction of matrotrophy is mapped onto the phylogeny, with blue colors

477 depicting lecithotrophy and orange colors matrotrophy (as indicated by the color scale of ln-

478 transformed matrotrophy index values). B. Frequency histogram of the distribution of matrotrophy

479 index values in extant poeciliid species. The solid red line represents the average ancestral state

480 reconstruction for the matrotrophy index across 250 random trees with dotted lines indicating the

$48195 \%$ confidence interval for the estimate. C. Frequency histogram depicting the relative change in

482 matrotrophy index between all nodes and their descendants. 

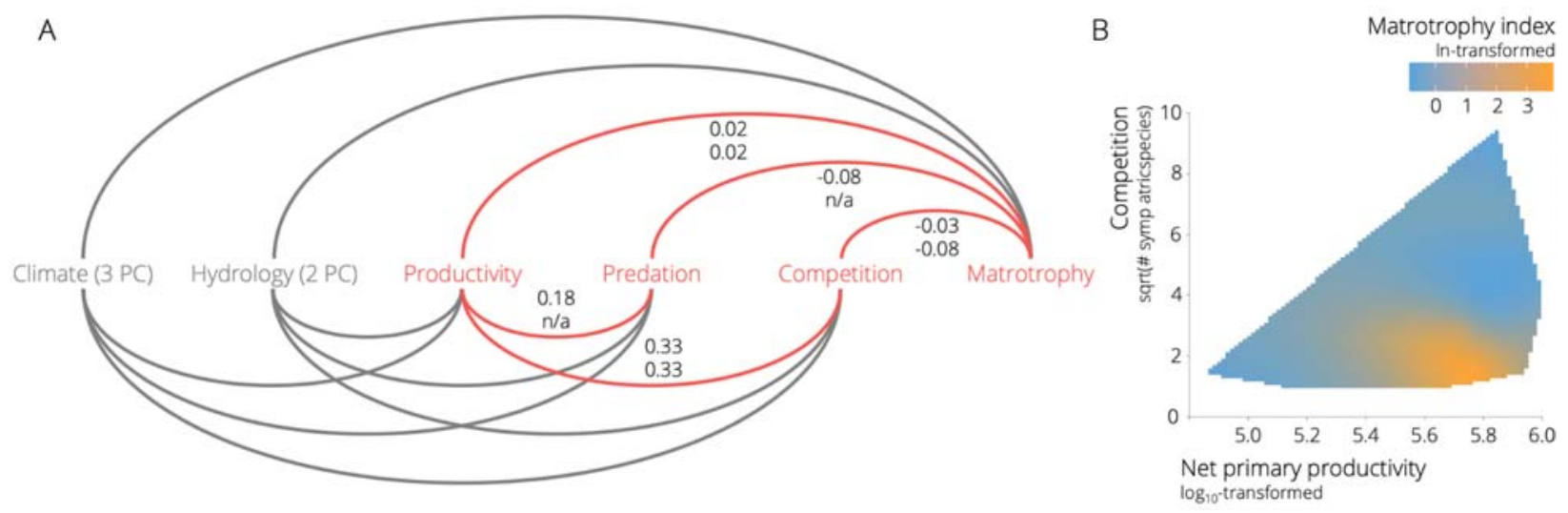

485 Figure 2. A. Representation of the full model used for phylogenetic path analysis (see Figure S2 for a

486 comprehensive depiction of alternative models). Highlighted in red are variables and paths

associated with the top models exhibiting $\Delta$ CIC $_{c}<2$. Numbers represent correlation coefficients $(r)$

488 between variables for the top model (top number) and the secondary model (bottom number). Note

489 that the factor predation was absent from the secondary model. B. Landscape of matrotrophy

490 variation as a function of net primary productivity and competition. Colors correspond to variation

491 in matrotrophy, with blue colors depicting maternal provisioning strategies toward the lecithotrophy

492 end of the spectrum and orange colors strategies toward the matrotrophy end (as indicated by the

493 color scale of ln-transformed matrotrophy index values). 